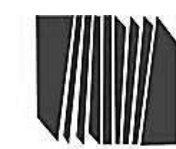

UNIVERSIDAD PEDAGOGICA EXPLRIMCNTAL LIOERTAOOR

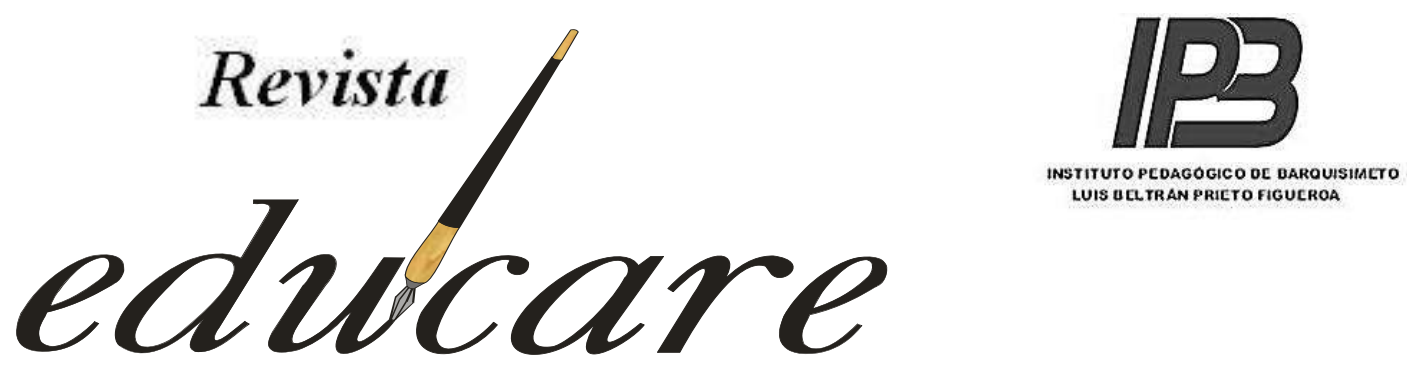

Órgano Divulgativo de la Subdirección de Investigación y Postgrado del Instituto Pedagógico de Barquisimeto "Luis Beltrán Prieto Figueroa"

\title{
PROCESO DE ACOMPAÑAMIENTO EN INVESTIGACIÓN EN EL NIVEL DE PREGRADO DESDE LA EXPERIENCIA DE TUTORES ACADÉMICOS
}

\author{
ACCOMPANYING PROCESS IN RESEARCH IN UNDERGRADUATE LEVEL FROM \\ THE EXPERIENCE OF ACADEMIC TUTORS
}

DEPOSITO LEGAL: ppi201002LA3674 ISSN: 2244-7296

\author{
Maricarmen Gamero*
}

\section{UNIVERSIDAD NACIONAL EXPERIMENTAL “FRANCISCO DE MIRANDA" (UNEFM)}




\begin{tabular}{|c|c|}
\hline TRABAJO DE INVESTIGACIÒN & Maricarmen Gamero ${ }^{*}$ \\
\hline Recibido: 19-01-2016 & Aceptado: 15-06-2016 \\
\hline RESUMEN & ABSTRACT \\
\hline $\begin{array}{l}\text { El presente artículo corresponde a un informe } \\
\text { de investigación cualitativa, enfocado en el } \\
\text { proceso de acompañamiento en investigación, el } \\
\text { cual atañe a las acciones, tanto de orientación, } \\
\text { mediación y entrenamiento en investigación, } \\
\text { como a las de apoyo psicosocial, liderado por un } \\
\text { tutor, quien atiende a las características y } \\
\text { necesidades académicas y personales de cada } \\
\text { tutorado dentro de un marco institucional } \\
\text { definido. La intencionalidad de la investigación } \\
\text { fue develar la actuación de tutores académicos } \\
\text { durante dicho proceso desde su propia } \\
\text { experiencia en el nivel de pregrado. Se empleó un } \\
\text { enfoque introspectivo-vivencial; en este sentido, } \\
\text { el diseño se enmarcó en la etnometodología a la } \\
\text { par que la estrategia para el acopio de } \\
\text { información fue la observación participante. Los } \\
\text { hallazgos muestran que las sesiones de } \\
\text { acompañamiento se llevan a cabo en tres fases: } \\
\text { preámbulo, abordaje del objeto de estudio y } \\
\text { cierre, realizadas con base en la producción de } \\
\text { contenido que haya realizado el tutorado. }\end{array}$ & $\begin{array}{l}\text { This article corresponds to a qualitative } \\
\text { research report, focused on the process of } \\
\text { research accompaniment, which corresponds to } \\
\text { the actions, in orientation, mediation and } \\
\text { research training, as well as psychosocial } \\
\text { support, led by a tutor, who attends to the } \\
\text { characteristics and academic and personal needs } \\
\text { of each tutor within a defined institutional } \\
\text { framework. The intention of the research was to } \\
\text { unveil the performance of academic tutors } \\
\text { during this process from their own experience at } \\
\text { the undergraduate level. An introspective- } \\
\text { experiential approach was used; In this sense, } \\
\text { the design was framed in ethnomethodology } \\
\text { while the strategy for gathering information was } \\
\text { participant observation. The findings show that } \\
\text { the accompaniment sessions are carried out in } \\
\text { three phases: preamble, approach to the object } \\
\text { of study and closure, carried out based on the } \\
\text { tutor's production of content. }\end{array}$ \\
\hline $\begin{array}{l}\text { Descriptores: tutores, } \quad \text { investigación, } \\
\text { acompañamiento }\end{array}$ & Keywords: tutors, research, accompaniment \\
\hline
\end{tabular}

* Maricarmen Gamero. Lcda. En Educación en Lengua Extranjera, Mención Inglés (UNEFM). MSc. en Investigación Educativa (Universidad de Carabobo). Msc. en Educación a Distancia (Universidad Internacional del Caribe)| Correo electrónico: gamero26@hotmail.com. 


\section{INTRODUCCIÓN}

Una de las actividades que propician el desarrollo óptimo de un país, proviene de los resultados o hallazgos que se obtengan a través de la realización de investigaciones científicas; por lo que las instituciones de educación universitaria, por ser las encargadas de formar profesionales que respondan a las demandas y necesidades de la sociedad, se constituyen en entes generadores de conocimiento.

En este sentido, la formación de investigadores y la producción científica estarían dentro de sus propósitos académicos; y es por ello que el estatus ideal de una universidad es que cuente con diferentes centros de investigación, promueva la actividad investigativa como un eje transversal durante todas las carreras que se impartan en ella, contando con la infraestructura, normas y bienes muebles necesarios para llevar a cabo disertaciones que contribuyan a ampliar las distintas ramas del saber humano.

En el caso de la Universidad Nacional Experimental "Francisco de Miranda" (UNEFM) de Coro- estado Falcón, la tarea científica se ha instituido como requisito para la obtención de ascensos del personal docente y de títulos académicos de pregrado y postgrado. Sin embargo, no existe una política uniforme en cuanto a la investigación; sino que corresponde a cada área académica y/o programa, la formulación de normas para el cumplimiento de tales requerimientos.

Un ejemplo de ello, es el Área de Educación, específicamente en el Programa de Educación, en el que se establece que los estudiantes en las menciones que lo conforman (Lengua, Literatura y Latín; Inglés; Informática; Música y Educación Física, Recreación y Deportes) deben entregar un trabajo especial de grado al finalizar su carrera y que cumpla con los criterios de calidad y pertinencia social. Por lo general, dicho producto es iniciado en los últimos trimestres de las carreras, a partir de la Unidad Curricular (U.C.) Seminario de Grado, lo que dispone un periodo relativamente breve para el desarrollo de competencias tan complejas como las de investigación.

Como tutora y evaluadora de trabajos de grado en el Programa de Educación por la mención Inglés, así como también ex miembro de la Coordinación de Trabajo de Grado en el Área de Educación de la UNEFM, la autora de esta investigación ha vivenciado situaciones en las que los estudiantes manifiestan dificultades en cuanto a la aplicación de sus conocimientos, habilidades y actitudes durante el desarrollo de sus investigaciones. También se ha logrado 
percibir casos en los que los estudiantes desarrollan eficientemente sus competencias en investigación, y prosiguen con su actividad científica una vez egresados de esta casa de estudios.

Ante esta divergencia de situaciones y tomando en cuenta que el investigador novel pertenece a un grupo de trabajo con un profesor guía o mentor, es imposible dejar de lado la labor del tutor, pues los logros alcanzados por sus tutorados son en parte representación de su capacitación como tutor y cumplimiento de sus funciones en el direccionamiento del trabajo de grado presentado. De allí que se consideró necesario tratar de develar la actuación de tutores académicos durante el proceso de acompañamiento en investigación llevado a cabo por los mismos, desde sus experiencias inmediatas; es decir, qué hacen y cómo llevan a cabo dicho proceso.

El proceso de acompañamiento se refiere al conjunto de acciones planteadas por los profesores, en conjunto con sus estudiantes y la institución, que comprende actividades de orientación académica, científica y personal (López, Dir., 2008). Si se proyecta dicha definición al ámbito de investigación, se puede decir que la misma se referiría a la ejecución y puesta en práctica de las funciones del tutor que definen su rol durante el desarrollo del estudio científico de sus tutorados, en concordancia con un marco institucional preestablecido, que en el caso de la presente investigación fue la Universidad Nacional Experimental "Francisco de Miranda".

A lo anteriormente mencionado, vale acotar que en el artículo 15 de las Normas de Trabajo de Grado del Programa de Educación, se señala que el tutor es responsable de formar y asesorar al estudiante de pregrado en el desarrollo de las habilidades exigidas en el proceso de investigación, organizar junto con su tutorado el plan de trabajo, llevar registro del mismo y evaluar periódicamente los avances del trabajo (UNEFM, 2009). En este sentido, el tutor académico estaría presente brindando el necesario acompañamiento a sus tutorados y cumpliendo sus funciones de orientación, mediación y entrenamiento necesario a los investigadores noveles.

Generalmente cuando los estudiantes del Programa de Educación inician sus trabajos de grado, es la primera vez que realizan formalmente investigaciones científicas en su carrera; por lo que la presencia y acompañamiento del tutor es de suma importancia para generar y hacer seguimiento al estudio realizado y a las actividades de sus tutorados. Esto se encuentra definido en las Normas de Trabajo de Grado del Programa de Educación como los deberes y derechos del tutor; sin embargo, al no establecerse un sistema de seguimiento, cada individuo cumpliría su función de acuerdo con su experiencia como estudiantes, de acuerdo con su ética o con lo que 
considera se encuentra dentro del parámetro de justicia. Por lo que tal situación podría acarrear algunas consecuencias satisfactorias y otras desfavorables en las que los tutores no intervienen en el desarrollo de la investigación y sólo ejercen su función para señalar la adecuación de la misma sin una revisión previa.

Los inconvenientes que se produzcan en las labores de los tutores durante el proceso de acompañamiento podrían tener repercusión en los estudiantes que se inician como investigadores y pueden comenzar a producirse cambios en algunas variables motivacionales; por ejemplo, baja autoestima académica, frustración, desinterés en la prosecución de metas, y apatía hacia la realización de investigaciones científicas, entre otros aspectos que conllevarían a actos antiéticos como el plagio, o darían origen al Síndrome Todo Menos Tesis (TMT). De esa manera se afectaría el perfil de egreso de los Licenciados en Educación de las diferentes menciones del Programa de Educación de la UNEFM; y por ende, la contribución que ellos podrían brindar al estado del arte de las ciencias educativas.

De lo anterior surgió la inquietud de entender cómo los tutores acompañan a sus tutorados durante el desarrollo de su investigación en el nivel de pregrado y el por qué lo hacen de tal manera, considerando su interacción con dichos estudiantes y el contexto situacional donde se desenvuelve tal proceso. En este sentido, el interés de la investigadora y el propósito del presente estudio científico era develar la actuación de tutores académicos durante el acompañamiento en investigación desde su propia experiencia en el nivel de pregrado. Por lo tanto, ontológicamente la investigación se enmarca en una realidad social sistemática construida por sus propios miembros, en la cual para poder comprender a fondo la naturaleza y el proceso del fenómeno se consideró la relación de los tutores con sus tutorados y su entorno, sus acciones y las circunstancias prácticas en las que se desenvuelven (Albert, 2007 y Martínez, 2006).

\section{ARGUMENTACIÓN TEÓRICA}

La universidad constituye el espacio en el que confluyen diversidad de pensamientos y culturas y donde se gestaría la creación y actualización de conocimientos de acuerdo con las demandas que emerjan conforme el paso del tiempo en la sociedad. Es por ello, que se considera que la detección y el medio para cumplir con el fin anterior requieren de la investigación científica, la cual ha sido convertida como requisito fundamental para obtener un grado 
académico o ascenso en el ámbito educativo.

$\mathrm{Al}$ producirse conocimiento en las universidades, independientemente de las razones de su creación (obtención de título, promoción de docentes, entre otros), brinda nuevos esquemas al estado del saber de un área académica específica. Ejemplos de ello pueden ser los trabajos de grado elaborados por los estudiantes del Programa de Educación de la UNEFM, que como estudio científico-técnico ha de tener pertinencia social, atender a las necesidades de la sociedad y contribuir con su transformación. De acuerdo con las vivencias de la autora en este proceso, cuando los investigadores son inexpertos y/o novatos, la presencia del tutor académico es esencial en la realización del producto científico y se constituye en fiel ejemplo y guía del proceso científico en la continua búsqueda de la verdad. Es aquí donde la interacción entre el tutorado y su mentor se configura como el proceso de acompañamiento en investigación.

\section{El Proceso de Acompañamiento en Investigación}

En la búsqueda bibliográfica que se llevó a cabo sobre el proceso de acompañamiento, se pudo apreciar que el mismo corresponde a las definiciones de tutoría y acción tutorial, haciéndolo el equivalente a tales actividades. Es decir que no existe un concepto preciso de este término; sin embargo, a partir de la experiencia de la investigadora como tutora y con ayuda de las ideas pertenecientes a distintos autores se puede expresar lo que esto implica. Generalmente las definiciones de tutoría y acción tutorial encontradas suelen corresponder a niveles educativos diferentes al de educación universitaria y a temas diferentes al ámbito de investigación; por lo que se trató de situar la definición del proceso de acompañamiento en el contexto de desarrollo de trabajos de grado en el nivel de pregrado.

En primer lugar se hace necesario saber qué es la tutoría, y en este caso González y Romo (2006) y Viel (2009) consideran que la misma es un proceso continuo de acompañamiento en el abordaje de aspectos académicos y personales que se centra en las necesidades, intereses e inquietudes del estudiantado de manera individual o grupal, para potenciar el desarrollo de habilidades de estudio o solucionar problemas educativos. Por su parte, Ubero (2010) plantea que la acción tutorial es un instrumento de asesoramiento o tratamiento personalizado que lleva a cabo el tutor, en conjunto con sus tutorados, mediante un plan de ejecución; para resolver inconvenientes de índole académico que pudieran atrasar la integración del estudiante a un nuevo contexto escolar o el logro de un mejor rendimiento académico. 
En este sentido, se puede concretar que el proceso de acompañamiento en investigación corresponde al conjunto de acciones, tanto de mediación, entrenamiento en investigación y orientación, como de apoyo emocional, liderado por un tutor quien atiende a las características y necesidades académicas y personales propias de cada tutorado a su cargo y dentro de un marco institucional definido. Todo ello, direccionado a la culminación exitosa de una investigación científica y la formación de habilidades en investigadores noveles.

Por lo tanto, el proceso de acompañamiento en investigación dependería en gran medida de la responsabilidad del tutor en el cumplimiento de cada una de sus funciones y roles, pero también en el vínculo y la reciprocidad que dicho actor social establezca con su tutorado. Dicha relación se desarrollaría de manera continua, tal como Palella y Martins (2010) señalan, la misma “transita de un acercamiento inicial más bien tímido, al descubrimiento de la afinidad académica que hace surgir el sentido de equipo tutorado-tutor y, finalmente, a una correspondencia plena que apoya el desenvolvimiento de un individuo en todas sus dimensiones” (p. 256). Así es como se iría construyendo la realidad del proceso de acompañamiento en investigación, en el cual la interacción entre tales actores sociales y el contexto podrían modificar los significados y actuación del otro.

El éxito del proceso de acompañamiento no se mide en la presentación formal y escrita de un trabajo de grado adecuadamente completado, sino en el desarrollo de competencias investigativas de los tutorados y el incremento de su motivación e interés hacia la tarea científica y hacia la contribución que puedan brindar al crecimiento científico de su nación. Para lograrlo, Palella y Martins (2010) indican que el ambiente propicio para el acompañamiento en investigación se caracterizaría por la comunicación, confianza, comprensión y respeto entre tutor y tutorado, siendo el primero de ellos el encargado de (a) identificar las habilidades del tutorado en cuanto a investigación, así como las características del contexto en el que se desenvuelve; (b) identificar la meta a lograr; y (c) diseñar conjuntamente con el tutorado, un plan estratégico tomando en cuenta las fortalezas, oportunidades, debilidades y amenazas a las cuales es propenso el estudiante.

Aquí, es necesario recalcar que también es importante que el tutor sea beneficiado durante la realización de la investigación. No me refiero a ningún tipo de ganancia económica o de alguna mención honorífica, sino del desafío que pueda representar el estudio y el enriquecimiento de sus competencias como explorador de la verdad. Asimismo, que le permita desarrollar habilidades 
como asesor académico y aprender la metodología para ejecutar las sesiones de acompañamiento.

\section{El Tutor}

El tutor es el coprotagonista del desarrollo de investigaciones, tanto en el nivel de pregrado como de postgrado, ya que vivencia junto con su tutorado todas las etapas del estudio que se ejecuta. Se podría decir que es el director de la orquesta, también el capitán del barco, pues es él quien da orientaciones al investigador novel y lo convierte en artífice de conocimientos. Su actividad no sólo recae en el campo académico, sino también personal, pues se convierte en un amigo y colaborador del estudiante en la prosecución de una meta en común, la finalización de un estudio investigativo.

Ruiz (2006) define al tutor académico desde dos vertientes, una como investigador modelo y la otra como docente cuya responsabilidad principal es dirigir la actividad académica del estudiante en cuanto a la elaboración de su trabajo de grado. En este sentido, debido a la complejidad del proceso de acompañamiento en investigación y tomando en consideración lo planteado por dicho autor, sería ideal que los tutores del Programa de Educación en la UNEFM sean investigadores expertos en su campo de especialización, ya que eso enriquecería sus conocimientos para guiar a sus tutorados durante la prosecución de su investigación. Asimismo, el hecho de ser educadores, les permitiría organizar el proceso de manera tal que se potencien las cualidades y habilidades de sus tutorados.

En este sentido, es conveniente que el tutor cuente con una serie de características que le puedan permitir desenvolverse y estar consciente de la responsabilidad que asume. Palella y Martins (2010) y Ruiz (2006), mencionan algunos aspectos claves del tutor y que, por lo general, no están expuestos a la vista del público, sino que emergen durante el desarrollo del trabajo de grado y en interacción con sus tutorados: cualidades científicas (conocimientos); cualidades técnicas (habilidades), y cualidades humanas (actitudes). Las primeras se refieren a los atributos que delimitan el saber del tutor; usualmente representadas por un grado académico, conocimientos amplios y profundos del tema de investigación; y conocimientos de teorías modernas de aprendizaje para guiar el proceso de acompañamiento. Las segundas son definidas por la experiencia del tutor como investigador; habilidades y destrezas para trabajar con investigadores noveles; dominio del método tutorial; manejo del computador como auxiliar del investigador e incluso disponibilidad de tiempo. Y finalmente, las cualidades humanas 
comprendidas por motivación hacia la actividad instruccional; actitud flexible y abierta hacia el aprendizaje permanente; asertividad, autoconocimiento, liderazgo, autenticidad, responsabilidad y sociabilidad.

Tales cualidades le permitirían al tutor cumplir con sus funciones y roles. Entre las primeras se encuentran ser modelo como investigador; cumplir con sesiones de asesorías; mostrar apertura a nuevas experiencias, empatía, autoconfianza e interés por la investigación; ofrecer apoyo al tutorado al conocer sus inquietudes, temores, intereses, gustos y preferencias de los tutorados; ser sincero con el tutorado y ayudarlo a ser fuerte para enfrentar los problemas que se puedan presentar en el transcurso de la investigación (Palella y Martins, 2010 y Ruiz, 2006). Todas estas funciones constituyen los roles que cumplirían los tutores en algún momento del proceso de acompañamiento, entre ellas la de facilitador, orientador, mediador e investigador.

\section{El Tutorado}

Durante el proceso de acompañamiento en investigación, el tutorado sería representado por el estudiante en la última etapa de su carrera que estaría siendo formado como investigador en su campo de estudio por un tutor académico, quien a su vez atiende a sus necesidades y rasgos académicos y personales. Para Palella y Martins (2010) el tutorado es el tesista en el proceso de elaboración de un trabajo científico, a quien, a través de una serie de estrategias didácticas, se le motiva a activar su espíritu investigativo y a desarrollar sus capacidades como actor social crítico y reflexivo, autogestor de conocimiento, agente de cambio y transformación de la sociedad.

Los tutorados como investigadores noveles podrían requerir de la continua interacción con su tutor para sentirse apoyados y orientados en momentos de ansiedad, lo que haría necesario para tales actores entablar una relación de trabajo basada en la cordialidad y respeto mutuo, lo que quizás favorezca todo el proceso de acompañamiento y el intercambio de experiencias de aprendizaje. Así, tal como lo menciona Giménez (2011), el tutorado podría ser más receptivo a las sugerencias u observaciones de su tutor y más responsable de sus opiniones.

El tutor dirigiría el proceso de acompañamiento en investigación, pero las cualidades del tutorado potenciarían el desarrollo eficiente de su trabajo de grado. Por ello, Valarino (2000) y Ruiz (2006) estiman que el investigador novel requeriría de ciertas características para desempeñarse en la tarea investigativa. Las primeras se refieren a los procesos cognitivos que lleva a cabo para aprender, rememorar y usar información relacionada con su trabajo de grado. 
Las segundas concernientes a la motivación, actitud positiva y valoración de la tarea científica, entre otras. La tercera incluye habilidades de búsqueda y procesamiento de información, resolución de problemas y toma de decisiones; así como también, redacción de avances técnicos.

Desde la perspectiva de la autora, en toda relación humana existe el sentido de reciprocidad y el vínculo tutor-tutorado, no es la excepción. A pesar del protagonismo y compromiso del tutor en sus distintas funciones y roles para encaminar el proceso de acompañamiento en investigación, dicho actor social respondería al entorno y ejecutaría sus tareas de acuerdo con el significado consciente que le atribuye a las mismas y a su interacción con los demás y el contexto. Es decir que el actuar de los tutores podría coincidir con la actuación de su tutorado y las características del contexto académico.

\section{El Contexto Académico}

El proceso de acompañamiento en investigación se fundamenta en la interacción de distintos sujetos dentro de un contexto específico, representado en el ámbito académico por la institución en la cual se lleva a cabo el mismo. Las ideas de Ruiz (2006) refuerzan esta aseveración, ya que el mencionado autor indica que existen "un conjunto de recursos externos, que intervienen directa o indirectamente en el proceso de elaboración del TG, condicionando el desempeño académico del tesista" (p. 131).

Las universidades como organizaciones suelen estar conformadas por una serie de elementos que hacen vida dentro de ella y que, a través de su interacción podrían crear y mantener una cultura organizacional propia, que la haría distintiva del resto e impulsaría su labor investigativa. La productividad en investigación de una institución de educación universitaria, en este caso la UNEFM, estaría acorde al apoyo que brinda la institución para promover la tarea científica. En este sentido, la actuación del tutor y tutorado se expresaría quizás en concordancia con la interacción entre ellos, y con el contexto académico institucional.

Ruiz (2006) y Valarino (2000) señalan que para disminuir aspectos como bajo rendimiento académico, deserción, ausentismo laboral, desmotivación hacia la investigación, entre otras, le correspondería a la institución dar soporte tanto a tutores como a tutorados, a través de infraestructura de apoyo para la investigación; por ejemplo, bibliotecas, centros de documentación, facilidades de computación; apoyo del supervisor, en la que se incluyen financiamiento, incentivos, entrenamiento en las funciones, líneas de investigación establecidas, 
reconocimiento y evaluación de la función del supervisor; y apoyo institucional a la evaluación del trabajo y apoyo al investigador, que comprenden el lapsos de entrega de avances o versión final, criterios preestablecidos de evaluación; financiamiento, incentivos, grupos de autoayuda, asesoría psicológica y asignaturas preparatorias.

\section{ASPECTOS METODOLÓGICOS}

Este estudio es de naturaleza cualitativa ya que pretendía alcanzar una visión de cómo ocurre el fenómeno de estudio, sin intentar manipular o interrumpir el escenario al controlar influencias externas o a diseñar experimentos. Así, se consideró la realización de lo que denomina Mayan (2001) de una aproximación inductiva, donde las ideas o categorías emergen de la actuación y contexto de la realidad donde se desarrolla el fenómeno de estudio.

En el mismo orden de ideas, la investigación está fundamentada en el paradigma interpretativo, por lo que las bases teóricas ocuparon un rol secundario, sin asumirlas a priori ya que podían influir en mi interpretación y comprensión del proceso o fenómeno de estudio (Corbetta, 2007). La matriz epistémica es de tipo fenomenológica, ya que describo la naturaleza del fenómeno tal como se manifiesta en el día a día de los protagonistas (Bisquerra, 2004 y Leal, 2005).

Con respecto al tipo de investigación desarrollada, la misma corresponde a la Etnometodología ya que se resalta la manera cómo los actores sociales llevan a cabo sus actividades y le dan orden a ese aspecto de su vida académica de forma consciente; claramente, todo ello exteriorizado a través del discurso y el comportamiento humano. Garfinkel (2006), creador del método, lo expone como el estudio de las actividades que realizan los miembros de un grupo o sector, que pueden ser explicables racionalmente en sí mismas, logrado esto a través del reconocimiento de las circunstancias y acciones prácticas, así como también el razonamiento sociológico práctico.

\section{Diseño de la Investigación}

Para poder recopilar información valiosa que permitiera construir la realidad junto con los actores sociales claves (tutores) durante el proceso de acompañamiento en investigación en el Programa de Educación de la UNEFM, se empleó un diseño emergente, básicamente 
interpretativo- comprensivo, el cual se inició con una intención de investigación y un conjunto de informantes clave que fue delimitando durante el proceso continuo de recogida y análisis de información; es decir que el diseño fue inductivo, abierto, flexible, cíclico y emergente (Bisquerra, 2004 y Albert, 2007). Para el método etnometodológico se estableció un diseño siguiendo los momentos propuestos por Valles (1997). En este sentido, el mismo comprendió tres etapas:

\section{Antes de Acceder al Escenario}

El primer momento comprende las actividades correspondientes al planteamiento de los propósitos iniciales, las estrategias de entrada al escenario, la selección de estrategias metodológicas y la temporalización (Bisquerra, 2004). Con respecto a la estrategia de entrada al escenario, es importante recalcar que la autora de este artículo como profesora del Programa de Educación de la UNEFM, estableció contacto con profesores tutores que quisieran participar en el estudio. Asimismo, tal proceso por llevarse a cabo en un grupo cerrado de dos o tres personas y ser muy privado, se concibió la posibilidad de integrar a la investigadora al grupo ejerciendo el rol de consultora metodológica para ayudar en ciertos momentos de inquietudes del tutor o tutorados, sin llegar a sustituir la labor del primero.

\section{El Trabajo de Campo}

El segundo momento del diseño corresponde al trabajo de campo, donde se seleccionó definitivamente a los informantes claves, se recogió la información y reajustó la temporalización del acopio de la misma a un periodo de tres meses, debido al estudio focalizado realizado a cada caso.

2.1 Informantes Clave. En primer lugar es conveniente mencionar que los informantes primarios fueron los profesores de pregrado que estaban iniciando, ya habían iniciado o estaban culminando el proceso de acompañamiento con sus tutorados en el Programa de Educación de la UNEFM. Ahora bien, ¿por qué el Programa de Educación y no otro? Sencillamente porque durante cinco (5) años la investigadora de este estudio ha sido profesora en dicha área y conoce la seriedad y responsabilidad que radica en cumplir las funciones de tutora y los distintos roles que en diversos momentos se tienen que asumir al vivenciar situaciones problemáticas académicas y 
personales. Es por ello que a raíz de dicha experiencia y de lo observado en otros actores sociales, se construyó junto con la información aportada por los informantes clave la realidad del fenómeno en estudio.

Inicialmente los informantes clave fueron tres (3) tutoras académicas pertenecientes a tres de las menciones adscritas al programa: inglés; lengua, literatura y latín; y educación física, recreación y deporte, ya que todas ellas se rigen bajo las mismas normas de trabajo de grado. Aquí, se debe recalcar que los tutorados fueron además informantes secundarios, ya que a pesar de no ser entrevistados directamente, interactúan con el tutor y están involucrados en su labor durante el acompañamiento dentro del contexto institucional de la universidad; por lo tanto, se hace notar su presencia durante la aplicación de la observación participante en asesorías presenciales.

El análisis de la información se llevó a cabo de manera progresiva luego de haber realizado la observación participante de cada situación; así, se iba comparando y contrastando los hallazgos que se encontraban, de manera que posterior a los tres primeros casos y debido a la diversidad de categorías emergidas en cada uno, fue necesaria la inclusión de uno nuevo. Aquí se debe acotar que la recolección de información se detuvo cuando se alcanzó un punto de saturación de la información al aplicar la estrategia metodológica escogida; la cual se describe a continuación.

\subsection{Estrategias Metodológicas para la Recolección de Información:}

Considerando la naturaleza del proceso de acompañamiento en investigación como el fenómeno de estudio, se empleó una estrategia que permitiera recopilar la mayor cantidad de información posible y poder construir junto con los informantes clave la realidad del mismo y lograr su comprensión. La misma fue la observación participante, la cual consistió en la participación directa e inmediata de la investigadora al asumir el rol de asesora metodológica dentro del grupo tutorial. En este caso, la forma de observación participante fue de participación natural, ya que hubo interacción con el mismo grupo que se investigaba (Ander-Egg, 2001). También se realizaron notas de campo de registros abiertos sin categorías preestablecidas, para seleccionar conductas, acontecimientos y procesos que ocurrían en el contexto tutorial durante el acompañamiento (Rojas, 2010 y Bisquerra, 2004). Conjuntamente con todo lo anterior se emplearon recursos de soporte que permitieran conservar por mayor tiempo la información obtenida, como los grabadores de audio y video. 


\section{Al Retirarse del Escenario}

Finalmente, en el tercer momento se contempló la retirada del campo de estudio y la realización de las tareas de análisis e interpretación de la información suministrada por los actores sociales; así como también de la verificación de los criterios de rigor científico y consideraciones éticas de la investigación (Bisquerra, 2004 y Albert, 2007). Tomando en consideración la intencionalidad y directrices de la investigación, así como también la particularidad de la información recopilada a través del registro de diálogos en formato de audio y video producido durante las sesiones de acompañamiento, se llevó a cabo un análisis conversacional empleando una técnica de tratamiento manual que permitiera la inmersión total del investigador en el contenido recopilado, la revisión constante de las categorías emergentes y la abstracción y posterior reflexión de la naturaleza del fenómeno.

\section{PRESENTACIÓN DE LOS HALLAZGOS}

A partir de la concatenación de las categorías principales identificadas en cada caso estudiado, emergieron las fases del proceso de acompañamiento en investigación, las cuales constituyen el razonamiento sociológico práctico derivado de la actuación de los tutores académicos en interacción con sus tutorados. Tales fases muestran el orden seguido por los tutores en las sesiones de acompañamiento observadas, las cuales se organizan en preámbulo, abordaje del objeto de estudio y cierre.

\section{Preámbulo (P)}

El preámbulo corresponde a todas las acciones que realizan los tutores para preparar el abordaje de la actividad central de las sesiones del proceso de acompañamiento. Es decir, constituye la apertura al diálogo entre el tutor y el tutorado, en el que se realiza una introducción del punto a tratar o se crea un ambiente de confianza que promueva el intercambio de ideas entre ambos actores sociales. Dentro del preámbulo el tutor pueden realizar una serie de acciones que precedan al tópico central, con el objeto de acondicionar al tutorado sobre éste, o sencillamente para tratar de recordar los convenios hechos entre ambos en anteriores reuniones.

Un ejemplo de lo anteriormente mencionado es el tutor que trata de evocar observaciones previas, realizando una serie de preguntas para recordar material y correcciones pendientes. En 
este caso, dependiendo del tiempo que haya transcurrido entre un encuentro y otro, pueden emerger dudas sobre las observaciones que él/ella haya planteado con anterioridad, por lo que es el tutorado quien debe manejar todas las observaciones y cambios realizados a sugerencia del tutor desde el inicio de la relación tutorial.

Otras de las acciones que ejecuta el tutor durante el preámbulo, es la introducción de la temática de la investigación, la cual consiste en una acotación teórica relacionada con el objeto de estudio, valiéndose de material de lectura para reforzar u orientar teóricamente al tutorado con respecto al tema de investigación.

Aparte de la evocación de correcciones previas o la introducción teórica que puede realizar el tutor, también se abordan temas sobre experiencias académicas que le permiten al profesor derribar cualquier barrera emocional con su tutorado e involucrarse más con las actividades que éste realiza, sobre todo en aquellas unidades curriculares relacionadas con la investigación, como lo es Seminario de Grado. Dicha indagación se realiza sólo en forma de interés personal, ya que el tutor no realiza seguimiento al desenvolvimiento de su tutorado en dicha unidad curricular por el hecho de considerarla como un requisito de aprobación.

En resumen, el preámbulo en el proceso de acompañamiento constituye la fase en la que el tutor capta la atención del estudiante y estimula su interés hacia la interacción con su persona; además, se determina el ambiente emocional en el que se desarrollará la comunicación. Luego, una vez que ambos sujetos se sincronizan en tiempo y espacio, el tutor procede con el abordaje del objeto de estudio.

\section{Abordaje del Objeto de Estudio}

El abordaje del objeto de estudio constituye la fase central del proceso de acompañamiento en investigación; en ella los tutores realizan una revisión teórica y metodológica, bien sea de la totalidad del trabajo o del avance que los tutorados presenten al momento de la reunión; al mismo tiempo que efectúan observaciones de forma al contenido.

Esta fase puede variar de acuerdo con la etapa de desarrollo en la que se encuentre la disertación. Un ejemplo del abordaje del objeto de estudio cuando el proceso de acompañamiento en investigación está iniciando, o se lleva a cabo en sincronía con la U.C. Seminario de Grado, corresponde a una sesión centrada en el primer, segundo y tercer capítulo del anteproyecto a entregar en la mencionada asignatura. 
En este particular, se desarticula cada capítulo de la investigación para profundizar en la revisión y explicación de los apartados que lo constituyen. Se consideran las sugerencias del Profesor de Seminario de Grado, se revisan las observaciones planteadas anteriormente y la reubicación de los intereses de su tutorado como investigador novel. Independientemente del tipo de paradigma adoptado, se hace especial énfasis en los objetivos o propósitos de la investigación, los cuales tienden a tener el sello personal del tutor y provienen de su propia consideración, denotando su preocupación por idear el camino a seguir para la realización de la investigación y el interés de que la misma sea culminada con éxito.

A medida que el tutor explica todo lo que se estará ejecutando en el trabajo especial de grado, señala otros componentes del mismo que el tutorado debe corregir y finalizar; por ejemplo, acota las observaciones de fondo y forma, y el establecimiento de criterios para selección de información. Asimismo, facilita material de lectura, lo que denota cuán involucrado se siente con el estudio, hasta el punto de realizar búsquedas por sí mismo y compartirla con el tutorado, promoviendo así la lectura de información que oriente los pasos a seguir en el propio trabajo científico. Esto lo hace de manera tal que el investigador novel sienta que tiene libertad de elección en los estándares que definirán su objeto de estudio y haciendo un llamado de atención sobre la importancia del tiempo que se deje pasar o que se emplee en ello.

El tutor puede indicar también la necesidad de reestructurar los elementos que se mencionan en el planteamiento del problema. Así, se requiere la inclusión de otros aspectos, en conjunto con las evidencias que ya se poseen de la situación objeto de estudio. Dependiendo de la forma cómo se realicen dichas observaciones, puede influirse positivamente o negativamente el filtro afectivo del tutorado, el cual puede sentir que no cubre las expectativas de su mentor o no posee las habilidades necesarias para llevar a cabo las actividades asignadas.

En cuanto a la metodología que emplea el tutor en el abordaje del objeto de estudio en el segundo capítulo, la misma recae principalmente en la determinación de las variables, a propósito del establecimiento de los objetivos de investigación, y la explicación de pautas de los otros componentes que lo conforman. Todo ello solicitándose intervenciones del tutorado en las que reflexione sobre el objeto de estudio; reafirmando aquellos aspectos que constituirán la base de su investigación a partir del diagnóstico de la problemática. Esto involucra una participación rica en la experiencia vivida por el tutorado como investigador, lo que le permite aportar ideas con propiedad a las decisiones tomadas. 
En dicha interacción, el tutor abarca distintos puntos del estudio en su segundo capítulo sin seguir un orden específico. Lo que le permite enriquecer sus explicaciones a medida que rememora propiedades claves de los apartados que se han de incluir en el trabajo y reanudar la explicación de tópicos los cuales se creían concluidos. Sin embargo, el hecho de no presentar la información de manera organizada crea confusión en la información que capta el estudiante, lo que se evidencia en el planteamiento de dudas al final de la sesión sobre los mismos temas ya abordados.

El tutor hace énfasis en la lectura de información, ya que a medida que el estudiante va conociendo más sobre el tema de su trabajo especial de grado, va adquiriendo una mayor autonomía sobre el rumbo que tome su investigación y de los fundamentos que lo sustentan. Entonces, el tutor puede llegar a emplear referencias bibliográficas que constituyan un modelo de antecedente de la investigación de su tutorado para dar un ejemplo de los criterios de búsqueda de otros y estructurar las bases teóricas que formarán parte del segundo capítulo. En cuanto a esta última actividad, el tutor recomienda el orden de presentación del soporte teórico del estudio, aunque el tutorado tiene la última palabra a medida que realiza su función de investigador.

En el tercer capítulo de la fase inicial del estudio científico que elabora el estudiante, el tutor continúa tomando a los objetivos de investigación como principio para la descripción global de lo que se ejecutará en el mismo; es decir que son tales propósitos los que le sirven de guía al tutor para cohesionar cada uno de los capítulos del trabajo y que el mismo sea metodológicamente coherente. En primer lugar, se aborda lo relacionado con la clasificación del estudio de acuerdo con su nivel, tipo y diseño de investigación, considerando la finalidad principal que pretende lograr el tutorado.

$\mathrm{Al}$ igual que en las explicaciones que el tutor realiza de los capítulos anteriores, vuelve a sugerir material de lectura para darle sustento teórico a todo lo que se incluirá en el marco metodológico del trabajo. A continuación, prescribe los criterios para la selección de la muestra, específicamente justificando el número de sujetos, la manera cómo ubicarlos, las características que deberían poseer y considerando las posibles problemáticas que podrían surgir luego, al momento de su escogencia. Tal explicación de las técnicas e instrumentos se realiza siguiendo el orden de las actividades que el tutorado debe llevar a cabo para lograr cada objetivo específico de la investigación, acompañado de una ejemplificación mental de su organización.

Cuando el abordaje del objeto de estudio se hace durante la culminación del anteproyecto 
de investigación en la U.C. Seminario de Grado, se realiza una revisión global de todo el informe, donde se inicia realizando una revisión de los aspectos formales de la totalidad del informe escrito, específicamente en cuanto a redacción y ortografía, aunado a la amplitud del mismo. Entonces, durante tal proceso, el tutor verifica la formalidad de las citas empleadas y la manera cómo el tutorado las emplea en el texto para justificar la investigación.

$\mathrm{Al}$ igual que en los otros momentos del estudio en los que se realicen las sesiones de acompañamiento, el tutor promueve la búsqueda de información y a medida que realiza la lectura de la producción de su tutorado, encuentra información que concuerda con su criterio de calidad y eficiencia y realiza un reforzamiento positivo. Es decir que el tutor no sólo resalta las posibles deficiencias que pudiera presentar su tutorado al momento de llevar a cabo su trabajo especial de grado, sino que también reconoce el esfuerzo y el buen trabajo hecho, lo que puede motivarlo y reforzar su confianza en sí mismo.

Con respecto a los instrumentos de recolección de información, el tutor y tutorado realizan un trabajo grupal en la elaboración de los mismos; sin embargo, una nueva revisión le permite al profesor asegurar la organización y uso adecuado de las interrogantes y su concordancia con los propósitos de la investigación. Dicha colaboración entre ambas partes, también se evidencia en los acuerdos sobre el título de la investigación.

Debido al interés del tutorado por culminar su investigación, es él quien solicita información con respecto a los periodos de inscripción de su proyecto ante la Coordinación de Trabajo de Grado. El tutor, por la ambigüedad que considera existe en las nuevas normas, no tiene seguridad sobre los pasos a seguir para la presentación escrita y oral de la investigación, y por consiguiente, delega la responsabilidad a su tutorado de comunicarse con las autoridades pertinentes sobre tales asuntos; condicionando su aprobación de inscripción al perfeccionamiento del producto final.

El tutor se considera el responsable del perfeccionamiento de la investigación, por lo que puede redactar o modificar por decisión propia el informe escrito. Un ejemplo de ello son los objetivos de investigación, los cuales son continuamente revisados en cada una de las fases del proceso de acompañamiento. Cuando el tutor tiene dudas con respecto a algún aspecto teórico del tema de estudio, prefiere dejarse guiar por su instinto y olvida que su tutorado tiene o debería tener conocimientos que podrían ayudar a mejorar la investigación.

Cuando el abordaje del objeto de estudio se lleva a cabo en un trabajo especial de grado 
que está siendo finalizado, la revisión también se direcciona hacia los aspectos formales de la totalidad del mismo. Sin embargo, se considera importante el tiempo restante para culminar la investigación, por lo que el tutor puede hacer un llamado de atención por la pérdida del mismo. Es aquí donde se observa la influencia de elementos laborales y familiares que pueden contribuir a la postergación de la investigación. Así, se muestra el tipo de problemas o disyuntivas a las que tienen que enfrentarse los tutorados al momento de producir su trabajo especial de grado, cuando deben asumir la responsabilidad de tomar decisiones y darle prioridad a algunas áreas de su vida.

El tutor examina la exactitud en la descripción de las técnicas e instrumentos de recolección de la información incluidos en el informe, el cronograma de actividades y la descripción del escenario. Asociado a esto, el tutor cuestiona el motivo por el cual alguna de las tareas asignadas no fueron realizadas, a lo que explica las implicaciones de tales elementos en el informe, en caso de que el motivo por el cual los tutorados no los hicieron, es porque tengan dudas de cómo expresar sus ideas con respecto a ellos.

En este caso, las sesiones de acompañamiento son llevadas a cabo en espacios y horas no académicas; lo cual es un indicativo de cuán involucrada se encuentra el tutor con el desarrollo del estudio. La labor del tutor en esta etapa de la investigación, comprende además la revisión de las actas impresas para la presentación oral del proyecto final de la investigación; es decir, que el compromiso que adquiere con sus tutorados incluye la verificación del cumplimiento de requisitos administrativos que les facilite a los estudiantes optar por su grado académico y culminar su formación profesional.

Después del tutor haber revisado cómo se aborda el objeto de estudio en la investigación de sus tutorados, procede a llevar a cabo la tercera fase del proceso de acompañamiento en investigación, en la cual se concreta lo relacionado con el próximo paso que debe realizar el estudiante para continuar avanzado en el camino científico.

\section{Cierre}

El cierre constituye la fase de culminación de la sesión de acompañamiento en investigación; en ella, los tutores reafirman todo lo discutido durante la reunión con los tutorados con el objeto de fijar los aspectos del estudio más importantes que requieren atención. La fase de cierre permite verificar que los estudiantes hayan comprendido las explicaciones, pautas y sugerencias, evitando así cualquier desvío teórico y metodológico causado por omisiones o 
confusiones. Por lo tanto, los tutores pueden repetir información que hayan mencionado anteriormente durante el encuentro.

Dentro de esta fase el tutor también establece tareas, tanto para su tutorado como para sí mismo. Básicamente las actividades del estudiante consisten en corregir las observaciones hechas y continuar produciendo cada sección de su trabajo especial de grado. Por su parte, las obligaciones del tutor comprenden la revisión de material y la elaboración de cualquier aspecto de la investigación que ofrezca realizar, esto si se considera a sí mismo como coautor del estudio.

Con base en tales asignaciones se realizan las sesiones siguientes; por lo que su culminación es preponderante para acordar el día, lugar y hora del próximo encuentro. Es decir que el proceso de acompañamiento no se lleva a cabo a partir de un plan de trabajo definido o a un cronograma, sino que es la producción del estudiante lo que la define, haciendo que haya una determinación casual de las sesiones. Sólo cuando el tutor ofrece asesoría durante un periodo vacacional prolongado es que se establece un acuerdo sobre el horario de las reuniones, o cuando hay consciencia del compromiso de tutores y tutorados y su capacidad de realizar las actividades en un periodo determinado, es que se realiza un consenso sobre las mismas. Es entonces en la fase de cierre que el tutor hace la conexión entre una sesión y otra del proceso de acompañamiento en investigación, al abordar aspectos tanto de la reunión que se está desarrollando, como de la próxima. Es allí donde se hace un sumario de las observaciones realizadas y el plazo para efectuarlas.

\section{REFLEXIONES FINALES}

El proceso de acompañamiento en investigación se desarrolla en torno a la revisión de la producción escrita del estudio científico del tutorado; es decir que el plan de trabajo no se define al inicio del mismo, sino que se establece a medida que el aprendiz comunica a su tutor la culminación de algún avance de su disertación. Es por ello que la relación tutorial inicia al mismo tiempo que la U.C. Seminario de Grado o una vez culminada.

Igualmente, la determinación de expectativas y metas, así como del contrato de aprendizaje y compromiso entre los actores sociales involucrados quedan establecidos de manera implícita, asumiéndose que el alumno por ser el aspirante y el interesado en la rápida culminación de su trabajo especial de grado cumplirá con todos los plazos de realización de actividades, y el 
profesor mostrará el mismo desempeño académico que tiene cuando maneja alguna asignatura. Lo anterior se contrapone a lo planteado por Ruíz (2006) y Ubero (2010), quienes destacan la importancia de diseñar en conjunto con el estudiante, un plan estratégico para intervenir cualquier obstáculo que se pueda presentar y guiar la actuación de los tutorados en el logro de metas y submetas. Es decir que llevar a cabo el proceso de acompañamiento en investigación sin un plan definido podría demorar el desarrollo del estudio y desviar la función y labor de tutores y tutorados al momento de enfrentar dificultades que entorpezcan el mismo.

Las sesiones de acompañamiento, consistentes en las fases de preámbulo, abordaje del objeto de estudio y cierre, se enfocan en las observaciones que plasma el tutor, en las cuales puede realizar acotaciones teóricas, sugerencias de formato de presentación del informe escrito y elementos metodológicos. Por lo tanto, sólo se abordan aspectos académicos relacionados con el estudio, obviando la orientación emocional que requiera el tutorado de parte de su mentor. Contrario a la idea de Pallela y Martins (2010) y Ruíz (2006) de señalar como función del tutor la de apoyar al tutorado al conocer sus inquietudes, temores e intereses y ayudarlo a enfrentar los problemas que se puedan presentar en el transcurso de la investigación.

La búsqueda e interpretación de la información constituye un aspecto clave del proceso de acompañamiento en investigación, la cual es fomentada por el tutor para el esclarecimiento de las dudas del tutorado, así como también para la ubicación de la problemática o tema de estudio, ya que a medida que el estudiante va conociendo más sobre el mismo, va adquiriendo una mayor autonomía sobre el rumbo que tome su investigación y de los fundamentos que lo sustentan.

Finalmente, el proceso de acompañamiento en investigación puede considerarse un proceso de enseñanza y aprendizaje que no sólo se constituye en un beneficio para el desarrollo de habilidades en investigación por parte de los estudiantes de pregrado; sino para el afianzamiento de las que ya posee el tutor, el desarrollo de su capacidad crítica y el aprendizaje de nuevos aspectos teóricos relacionados con su especialidad. 


\section{REFERENCIAS}

Albert, M. (2007). La investigación educativa. Claves teóricas. Madrid: Mc Graw-Hill.

Ander-egg, E. (2001). Métodos y técnicas de investigación social. Buenos Aires: Editorial Lumen.

Bisquerra, R. (2004). Metodología de la investigación educativa. Barcelona: La Muralla.

Corbetta, P. (2007). Metodología y técnicas de investigación social. Edición revisada. Madrid: Mc Graw-Hill.

Garfinkel, H. (2006). Estudios en Etnometodología. (H. A. Pérez, Trad.). Barcelona: Anthropos Editorial. (Trabajo original publicado en 1968).

Giménez, J. (2011). La comunicación en el proceso de la acción tutorial. Valencia: Asociación de Profesores de la Universidad de Carabobo.

González, R. y Romo, A. (2006). Detrás del acompañamiento. ¿Una nueva cultura docente? Obtenido el20 de Enero de 2015 desde: http://books.google.co.ve/books?id=ZrW17oX_vkIC\&printsec=frontcover\&hl=es\&source=g bs_ge_summary_r\&cad $=0 \# v=$ onepage $\& q \& f=f a l s e$.

Leal, J. (2005). La autonomía del sujeto investigador y la metodología de la investigación. Mérida: Centro Editorial Litorama.

López, F. (Dir.). (2008). La acción tutorial: El alumnado toma la palabra. Caracas: Editorial Laboratorio Educativo.

Martínez, M. (2006). Ciencia y arte en la metodología cualitativa. México: Trillas.

Mayan, M. (2001). Una introducción a los métodos cualitativos: módulo de entrenamiento para estudiantes y profesionales. Alberta: QualInstitutePress..

Palella, S. y Martins, F. (2010). Metodología de la investigación cuantitativa. (3 ${ }^{\text {a }}$ Ed.). Caracas: FEDUPEL.

Rojas, B. (2010). Investigación cualitativa. Fundamentos y praxis. (2 ${ }^{\mathrm{a}}$. Ed.). Caracas: FEDUPEL.

Ruiz, C. (2006). Como llegar a ser un tutor competente. La tutoría de tesis de grado. Caracas: Aula XXI Santillana/UPEL.

Ubero, N. (Coord.). (2010). Formación, competitividad y empleo. Experiencias de adaptación al EEES en la facultad de biología. Obtenido el20 de Enero de 2015 desde:http://books.google.co.ve/books?id=8bxOCUZvA2oC\&printsec=frontcover\&hl=es\&s ource $=\mathrm{gbs} \_g e \_s u m m a r y \_r \& c a d=0 \# \mathrm{v}=$ onepage $\& \mathrm{q} \& \mathrm{f}=$ false.

Universidad Nacional Experimental "Francisco de Miranda", Comisión de Trabajo de Grado del Programa de Educación. (2009, Septiembre) Normas de trabajo de grado del programa de educación. Coro: Autor.

Valarino, E. (2000). Tesis a tiempo. Obtenido el20 de Enero de 2015 desde:http://books.google.co.ve/books?id=w_vA02P3obgC\&printsec=frontcover\&hl=es\&so urce $=$ gbs_ge_summary_r $\&$ cad $=0 \# \mathrm{v}=$ onepage $\& \mathrm{q} \& \mathrm{f}=\mathrm{false}$. 
Valles, M. (1997). Técnicas cualitativas de investigación social. Reflexión metodológica y práctica profesional. Madrid: Editorial Síntesis.

Viel, P. (2009). Gestión de la tutoría escolar. Obtenido el20 de Enero de 2015 desde: http://books.google.co.ve/books?id=pr-n695SQ0MC\&printsec=frontcover \&hl= es\&source= gbs_ge_summary_r\&cad=0\#v=onepage $\& q \& \mathrm{f}=$ false 